\title{
Introduction au numéro spécial du Bulletin de la SPE, consacré au suivi médico-anthropologique de personnes vivant avec le VIH (PvVIH) traitées par ARV à Dakar (1999-2010) (cohorte ANRS 1215)
}

\section{J.-F. Delfraissy \\ (C) Société de pathologie exotique et Springer-Verlag France 2014}

Sous l'impulsion de la politique d'accès universel promue par l'OMS à partir de 2003 et grâce aux fonds multilatéraux, le nombre de personnes recevant des traitements antirétroviraux dans les pays à ressources limitées s'est accru de manière spectaculaire ces dix dernières années. La généralisation progressive de la prise en charge thérapeutique, associée aux renforcements des efforts de prévention, se traduit par un net ralentissement de l'épidémie à l'échelle mondiale. En 2014, en Afrique, le nombre de nouvelles infections par le VIH a diminué ou s'est stabilisé dans trente-trois pays. Nous assistons enfin au reflux de l'épidémie.

La stratégie de généralisation de l'usage des antirétroviraux a été définie à partir d'un ensemble de connaissances scientifiques acquises à travers une dynamique de recherche internationale. L'ANRS y a largement contribué.

Actuellement, l'impact à court, moyen terme du traitement, tant au niveau biomédical que social, est bien documenté et l'accroissement récent du nombre de personnes recevant des traitements ARV a permis de consolider et d'affiner les connaissances initiales. En revanche, l'impact à long terme, individuel et collectif des ARV dans les pays à ressources limitées est source de nombreuses interrogations. Elles relèvent de plusieurs disciplines scientifiques.

En 1998, le Sénégal fut l'un des premiers pays d'Afrique à mettre en place un programme gouvernemental de traitement par les ARV (l'Initiative sénégalaise d'accès aux médicaments antirétroviraux - ISAARV), sous la direction de quelques personnalités pionnières - Pr Awa Coll-Seck, Pr Souleymane Mboup, Dr Ibra Ndoye, Pr Papa Salif Sow qui ont su porter une stratégie nationale audacieuse et innovante pour l'époque. Dés le début, l'ANRS a soutenu cette initiative à travers plusieurs programmes de recherches dont il a été rendu compte dans diverses publications internationales notamment un ouvrage édité par l'ANRS en 2002 puis avec l'OMS et l'ONUSIDA en 2004 [1].

Les recherches débutées en 1999 auprès des premiers patients traités à Dakar ont été poursuivies dans le cadre

J.-F. Delfraissy $(\bowtie)$

ANRS (France Recherche Nord \& Sud Sida-HIV Hépatites),

101 Rue de Tolbiac 75013, Paris

e-mail : jf.delfraissy@anrs.fr d'une cohorte observationnelle prospective - la cohorte ANRS 1215 - jusqu'en 2011, avec le soutien de l'ANRS et la participation de l'Institut de recherche pour le développement, du ministère français des Affaires Étrangères et de l'Union européenne. Coordonné par le Dr Ibra Ndoye (CNLS) et le Pr Eric Delaporte (IRD), puis le Dr Bernard Taverne (IRD), ce programme de recherche fut exemplaire à plusieurs titres : par son approche multidisciplinaire (virologie, épidémiologie, clinique, santé publique et sciences sociales), par sa perspective opérationnelle, par sa durée (12 ans) et par la relation de partenariat scientifique privilégié créée entre les équipes de cliniciens et chercheurs sénégalais et français.

Les articles rassemblés dans ce numéro du Bulletin de la Société de pathologie exotique présentent les principaux résultats de cette recherche. Après un état des connaissances sur l'impact à long terme des traitements ARV en Afrique, sont abordés successivement les aspects biologiques et épidémiologiques, l'expérience individuelle et collective de la maladie, les aspects sociaux relatifs aux soins, et enfin, le rôle du système de santé. La diversité des angles d'observation combinée à la précision des analyses offre ici une compréhension fine des divers enjeux liés aux traitements.

Je suis convaincu que les résultats de ces travaux publiés depuis 2000 ont participé à l'amélioration de l'organisation de la prise en charge médicale et sociale des personnes vivant avec le VIH dans les pays du Sud. Les données publiées dans ce numéro vont contribuer à ce que cette prise en charge s'améliore encore.

Je tiens à remercier le comité éditorial du Bulletin de la Société de pathologie exotique pour avoir coordonné la publication de ce numéro, et le féliciter pour son engagement et sa constance à la diffusion d'une information scientifique de haut niveau à destination des acteurs de santé de l'espace francophone.

\section{Référence}

1. Desclaux A, Lanièce I, Ndoye I, Taverne B (eds) (2002) L'Initiative sénégalaise d'accès aux médicaments antirétroviraux. Analyses économiques, sociales, comportementales et médicales. ANRS, UNAIDS, WHO, Paris, 2004, 230 p. 